\title{
○

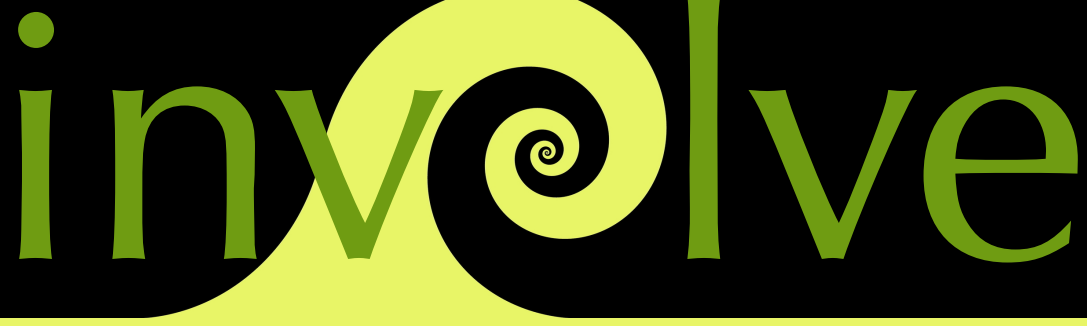 \\ a journal of mathematics
}

Rank disequilibrium in multiple-criteria evaluation schemes

Jonathan K. Hodge, Faye Sprague-Williams and Jamie Woelk 


\title{
Rank disequilibrium in multiple-criteria evaluation schemes
}

\author{
Jonathan K. Hodge, Faye Sprague-Williams and Jamie Woelk
}

(Communicated by Kenneth S. Berenhaut)

\begin{abstract}
In multiple-criteria evaluation schemes, rank disequilibrium occurs when an evaluee is rated higher than other evaluees on some criteria and lower than other evaluees on other criteria. In this article, we investigate rank disequilibrium as it relates to the problem of aggregating scores on individual criteria into an overall evaluation. We adopt an axiomatic approach, defining the notion of a rank aggregation function and proposing a set of desirable properties - namely, independence, monotonicity, inclusivity, consistency, and equity - that rank aggregation functions may or may not satisfy. We prove that when there are more than three possible scores on each criterion, it is impossible to define a rank aggregation function that satisfies all of these properties. We then investigate potential resolutions to the problems posed by rank disequilibrium.
\end{abstract}

\section{Introduction}

According to Pruitt and Kim [2004, p. 24], rank disequilibrium, or status inconsistency, "exists when there are multiple criteria for assessing people's merit or contributions, and some people are higher on one criterion and lower on another criterion than others". Status inconsistency has been studied at length within the sociology and conflict resolution literature, particularly in regards to social stratification, intergroup conflict, and aggression (for example, [Engel 1988; Evan and Simmons 1969; Galtung 1964; Hernes 1969; Kriesberg 1998; Muller and Jukam 1983; Segal et al. 1970]). This article focuses on the phenomenon of rank disequilibrium within the specific context of multiple-criteria evaluation.

MSC2010: primary 91B08, 91B14; secondary 06A07.

Keywords: rank disequilibrium, status inconsistency, inequity, multiple-criteria evaluation, rank

aggregation function.

This research was supported by the National Science Foundation under Grant No. DMS-1003993, which funds a Research Experiences for Undergraduates (REU) program at Grand Valley State University. Any opinions, findings and conclusions or recommendations expressed in this material are those of the author(s) and do not necessarily reflect the views of the National Science Foundation (NSF). 
To illustrate, consider the common practice of evaluating employees by first assigning scores on a variety of criteria and then determining an overall performance rating by aggregating these scores in some way. For example, it is common within academia for faculty to be evaluated on teaching, research, and service. But what happens when Professor Smith is rated satisfactory in teaching, outstanding in research, and outstanding in service, while Professor Jones is rated outstanding in teaching, satisfactory in research, and satisfactory in service? Who should receive a higher overall rating? On the one hand, Professor Smith could argue that she is entitled to the higher rating, since she received higher marks on two of the three criteria. On the other hand, Professor Jones could argue that teaching is the most important criterion, and so she should receive the higher rating. The inconsistency between each professor's scores on the various criteria presents challenges to the evaluator who must aggregate the scores and determine an overall evaluation. Indeed, it is conceivable that, regardless of the final evaluations, one of the two professors will perceive that she has been treated inequitably.

Much of the prior research on rank disequilibrium has been empirical or philosophical in nature. In the present work, we take an axiomatic approach. In Section 2, we introduce the definitions that form the basis of our model. We define the notion of a rank aggregation function, which is our primary mechanism for aggregating marks on individual criteria into a single, overall evaluation. We then propose several desirable properties of rank aggregation functions - including independence, monotonicity, inclusivity, consistency, and equity - giving examples to illustrate each. Rank aggregation functions that satisfy all of these properties are said to be ideal.

In Sections 3 and 4, we consider conditions for the existence and uniqueness of ideal rank aggregation functions. We demonstrate the existence of a unique ideal rank aggregation function in the case where only three scores are possible for each criterion. We then prove that when more than three scores are allowed, no such function exists.

In Sections 5 and 6, we consider potential resolutions to the nonexistence (in most cases) of ideal rank aggregation functions. We show that suitable alternatives can be found if we are willing to sacrifice independence or accept a weaker form of equity.

In Section 7, we summarize our work and discuss questions for further research.

\section{Definitions and examples}

Our model assumes that a finite number of evaluees will receive one of a finite number of ratings, or scores, on each of a finite number of criteria.

We let $C$ denote the set of evaluation criteria, where $|C|=n$. We let $Z=$ $\left\{z_{1}, z_{2}, \ldots, z_{m}\right\}$ denote the set of possible scores for each criterion, where $m \geq 2$. 
We assume that $Z$ is totally ordered, with the ordering relation denoted by $\succeq$, where $z_{m} \succ z_{m-1} \succ \cdots \succ z_{2} \succ z_{1}$. (Intuitively, $z_{m}$ corresponds to the best score and $z_{1}$ corresponds to the worst score.)

A score profile, or profile for short, is an $n$-tuple of elements of $Z$ - that is, an element of the Cartesian product

$$
X=Z \times Z \times \cdots \times Z=Z^{n} .
$$

This set $X$ is called the profile space. For any profile $x \in X$, we let $x_{c}$ denote the score from the $c$-th criterion (coordinate) of $x$. If, for some $x, y \in X$, we have $x_{c} \succeq y_{c}$ for all $c \in C$, then $x$ is said to dominate $y$, denoted $x \gg y$. For any $x \in X$, we define $\min x$ and $\max x$ in a natural way - namely,

$$
\min x=\min _{c \in C}\left\{x_{c}\right\} \quad \text { and } \max x=\max _{c \in C}\left\{x_{c}\right\},
$$

where the notions of minimum and maximum are defined with respect to the total order $\succeq$ on $Z$.

We use the notation $\vec{z}$ to denote the profile $x$ for which $x_{c}=z$ for all $c \in C$. Such a profile is called a uniform profile. For every nonempty proper subset $R$ of $C$, let $X_{R}=Z^{|R|}$ and $X_{-R}=Z^{n-|R|}$. For all $x \in X_{R}$ and $y \in X_{-R}$, we denote by $(x, y)$ the profile that coincides with $x$ for the criteria in $R$ and coincides with $y$ for the criteria not in $R$. In other words, $(x, y)_{c}=x_{c}$ for all $c \in R$ and $(x, y)_{c}=y_{c}$ for all $c \notin R$. To denote the restriction of a uniform profile $\vec{z}$ to the criteria in $R$, we write $\vec{z}_{R}$.

An evaluee $e$ is an ordered pair $\left(x^{e}, \succeq_{e}\right)$, where $x^{e} \in X$ and $\succeq_{e}$ is a monotone weak order on $X$ - that is, a weak order in which $x \succeq_{e} y$ whenever $x \gg y$. The first coordinate, $x^{e}$, represents the vector of scores assigned to $e$ by the evaluation process (one for each evaluation criterion). The second coordinate, $\succeq_{e}$, represents $e$ 's perceived ordering of the possible profiles according to their relative value or desirability. (We will call such an ordering a profile ordering.) Monotonicity imposes a minimal assumption of rationality on each evaluee's profile ordering for example, by prohibiting an evaluee from viewing a rating that is unsatisfactory in every category as more desirable than one that is satisfactory in every category. However if $x$ and $y$ are two profiles with the property that $x \ngtr y$ and $y \ngtr x$ (in which case $x_{i} \succ y_{i}$ and $y_{j} \succ x_{j}$ for some $i \neq j$ ), then the monotonicity condition imposes no restrictions on an evaluee's relative ordering of $x$ and $y$. In this case, one can posit the existence of evaluees $e_{1}$ and $e_{2}$ for which $x \succ_{e_{1}} y$ and $y \succ_{e_{2}} x$.

The set of all possible evaluees is called the evaluee space and denoted $\mathcal{E}$. The set of all submultisets of $\mathcal{E}$ is denoted $\mathcal{P}(\mathcal{E}){ }^{1}$

\footnotetext{
${ }^{1}$ Formally, our definitions must involve multisets to allow for the possibility of multiple evaluees having the same profile and profile ordering. However, from this point forward, we will, for convenience, use the simpler language of sets and subsets, keeping in mind that our results apply to (and sometimes require) multisets as well.
} 
Definition 2.1. A rank aggregation function is a function that maps each pair $(x, E)$, where $x$ is a profile and $E$ is a subset of $\mathcal{E}$, to an element of $Z$. In other words, a rank aggregation function is a mapping $f: X \times \mathcal{P}(\mathcal{E}) \rightarrow Z$.

For any rank aggregation function $f$, we will use the notation $f_{E}(x)$ to denote $f(x, E)$. This notation captures the idea that, for a fixed set of evaluees, we can view $f$ as nothing more than a function on the profile space $X$. By definition, the function $f$ is anonymous: it assigns the same overall score to any two evaluees who have the same profile. However, $f$ may take into account information about the set of evaluees as a whole - including evaluees' profile orderings - so that if this information changes in any way, the scores assigned by $f$ may also change. A rank aggregation function that assigns the same scores regardless of the set of evaluees is said to be independent. The next definition formalizes this idea and defines several additional desirable properties that a rank aggregation function might satisfy.

Definition 2.2. Let $f: X \times \mathcal{P}(\mathcal{E}) \rightarrow Z$ be a rank aggregation function. Then:

(i) $f$ is independent, provided that $f_{E_{1}}(x)=f_{E_{2}}(x)$ for all $E_{1}, E_{2} \in \mathcal{P}(\mathcal{E})$ and all $x \in X$.

(ii) $f$ is monotone, provided that $x \gg y$ implies $f_{E}(x) \succeq f_{E}(y)$ for all $x, y \in X$ and all $E \in \mathcal{P}(\mathcal{E})$.

(iii) $f$ is inclusive, provided that for each nonempty, proper subset $R$ of $C$, there exists $E \in \mathcal{P}(\mathcal{E})$ and profiles $(x, u),(y, u)$, where $x, y \in X_{R}$ and $u \in X_{-R}$, such that $f_{E}(x, u) \neq f_{E}(y, u)$. (In this case, $C$ is said to be minimal with respect to $f$.)

(iv) $f$ is consistent with respect to $E \in \mathcal{P}(\mathcal{E})$, provided that $f_{E}(\vec{z})=z$ for all $z \in Z$. If $f$ is consistent with respect to all $E \in \mathcal{P}(\mathcal{E})$, then $f$ is said to be universally consistent, or simply consistent.

(v) $f$ is equitable with respect to $E \in \mathcal{P}(\mathcal{E})$, provided that $x \succ_{e} y$ implies $f_{E}(x) \succeq$ $f_{E}(y)$ for all $e \in E$ and all $x, y \in X$. (If, for some evaluee $e \in E, x \succ_{e} y$ and $f_{E}(y) \succ f_{E}(x)$, then $e$ is said to perceive inequity.) If $f$ is equitable with respect to all $E \in \mathcal{P}(\mathcal{E})$, then $f$ is said to be universally equitable, or simply equitable.

A rank aggregation function that is independent, monotone, inclusive, consistent, and equitable is said to be ideal. If $f$ is an independent rank aggregation function, then we will denote by $f(x)$ the common value of $f_{E}(x)$ for all $E \in \mathcal{P}(\mathcal{E})$.

Example 2.3. Let $f$ be the rank aggregation function defined by $f(x)=x_{1}$. (Note that $f$ assigns to each profile the score received on the first criterion.)

By definition, $f$ is independent and consistent. Furthermore, $f$ is monotone since $x \gg y$ implies $x_{1} \succeq y_{1}$.

Since $f$ assigns scores based solely on the first criterion of each profile, it is intuitively obvious that $f$ is not inclusive. To prove this formally, let $R$ be any 
nonempty, proper subset of $C$ that does not include the first criterion. Then

$$
f(x, u)=(x, u)_{1}=u_{1}=(y, u)_{1}=f(y, u)
$$

for all $x, y \in X_{R}$ and all $u \in X_{-R}$. So $C$ is not minimal, and $f$ is not inclusive.

Finally, we will show that $f$ is not universally equitable. Let $E$ be any set of evaluees containing an evaluee $e$ for which

$$
\left(z_{1}, z_{m}, \ldots, z_{m}\right) \succ_{e}\left(z_{m}, z_{1}, \ldots, z_{1}\right) .
$$

Such an evaluee exists since

$$
\left(z_{1}, z_{m}, \ldots, z_{m}\right) \ngtr\left(z_{m}, z_{1}, \ldots, z_{1}\right) \text { and }\left(z_{m}, z_{1}, \ldots, z_{1}\right) \gg\left(z_{1}, z_{m}, \ldots, z_{m}\right) \text {. }
$$

Note, however, that since

$$
f\left(z_{m}, z_{1}, \ldots, z_{1}\right)=z_{m} \succ z_{1}=f\left(z_{1}, z_{m}, \ldots, z_{m}\right),
$$

it follows that $e$ perceives inequity, and $f$ is not equitable with respect to $E$.

In conclusion, we have shown that $f$ is independent, monotone, and consistent, but neither inclusive nor equitable.

Example 2.4. Let $f$ be the rank aggregation function defined by $f(x)=\max x$.

By definition, $f$ is independent and consistent. We will show that $f$ is inclusive and monotone, and $f$ is equitable if and only if $m=2$.

For inclusivity, let $R$ be any proper, nonempty subset of $C$. Then

$$
f\left(\vec{z}_{1}\right)=f\left(\left(\vec{z}_{1}\right)_{R},\left(\vec{z}_{1}\right)_{-R}\right)=z_{1},
$$

but

$$
f\left(\left(\vec{z}_{m}\right)_{R},\left(\vec{z}_{1}\right)_{-R}\right)=z_{m} .
$$

Since $f$ assigns different scores to two profiles that differ only on $R$, and $R$ was chosen arbitrarily, it follows that $C$ is minimal with respect to $f$. Thus, $f$ is inclusive.

For monotonicity, let $x, y \in X$ such that $x \gg y$. Then $x_{c} \succeq y_{c}$ for all $c \in C$, which implies

$$
f(x)=\max x \succeq \max y=f(y) .
$$

Thus, $f$ is monotone.

For equity, note that if $m \geq 3$, any evaluee $e$ for which

$$
\vec{z}_{2}=\left(z_{2}, z_{2}, \ldots, z_{2}\right) \succ_{e}\left(z_{1}, z_{1}, \ldots, z_{1}, z_{m}\right)
$$

will perceive inequity, since

$$
f\left(z_{1}, z_{1}, \ldots, z_{1}, z_{m}\right)=z_{m} \succ z_{2}=f\left(\vec{z}_{2}\right) .
$$

(Intuitively, such an evaluee views a profile that receives the second worst score on each criterion as favorable to one that receives the worst score on all but one 
of the criteria and the best score on the remaining criterion.) This example clearly breaks down if $m=2$. Note that for an evaluee $e$ to perceive inequity, it must be that, for some profiles $x$ and $y$, we have $x \succ_{e} y$ and $f(y) \succ f(x)$. But when $m=2$, $f(y) \succ f(x)$ only if $f(y)=z_{2}$ and $f(x)=z_{1}$. This, however, implies $x=\vec{z}_{1}$, a contradiction to the monotonicity of $\succ_{e}$ in light of the assumption that $x \succ_{e} y$.

To summarize, we have shown that $f$ is independent, monotone, consistent, and inclusive. In the case that $m=2$ (and only in this case), $f$ is also equitable, and thus ideal.

Example 2.5. For a more concrete example, consider an evaluation process with three evaluation criteria $(n=3)$ and three possible scores $(m=3)$ for each criterion:

$$
\text { outstanding } \succ \text { satisfactory } \succ \text { unsatisfactory. }
$$

Define a rank aggregation function $f$ as follows (abbreviating each score by its first letter):

$$
f_{E}(x)= \begin{cases}O & \text { if } x \succ_{e} x^{e} \text { for all } e \in E \text { with } x^{e} \neq x \\ U & \text { if } x^{e} \succ_{e} x \text { for all } e \in E \text { with } x^{e} \neq x \\ S & \text { otherwise. }\end{cases}
$$

Suppose Sally is being evaluated using $f$. Then the only way for Sally to be rated as outstanding is for every other evaluee whose profile is different from hers to view Sally's profile as the more favorable one. In essence, every evaluee must envy Sally's profile. Likewise, for Sally to be rated as unsatisfactory, every evaluee with a different profile than Sally's must view their own profile as favorable to hers.

Now consider the set $E$ consisting of three evaluees $-a, b$, and $c-$ for whom the following conditions hold:

- $x^{a}=(S, S, O)$ and $(S, S, O) \succ_{a}(S, O, S) \succ_{a}(O, S, S)$;

- $x^{b}=(S, O, S)$ and $(S, O, S) \succ_{b}(O, S, S) \succ_{b}(S, S, O)$;

- $x^{c}=(O, S, S)$ and $(O, S, S) \succ_{c}(S, S, O) \succ_{c}(S, O, S)$.

Note that, in this case, $f_{E}(S, S, O)=f_{E}(S, O, S)=f_{E}(O, S, S)=U$, since every evaluee favors their own profile over those of each of the other evaluees. (This is not a terribly surprising outcome, since evaluees tend to exhibit self-serving biases.)

Consider, however, another set $E^{\prime}$ of evaluees who, one might argue, are less tainted by their individual biases:

- $x^{u}=(S, S, O)$ and $(O, S, S) \succ_{u}(S, O, S) \succ_{u}(S, S, O)$;

- $x^{v}=(S, O, S)$ and $(O, S, S) \succ_{v}(S, O, S) \succ_{v}(S, S, O)$;

- $x^{w}=(O, S, S)$ and $(O, S, S) \succ_{w}(S, S, O) \succ_{w}(S, O, S)$. 
It is easy to verify that $f_{E^{\prime}}(S, S, O)=U, f_{E^{\prime}}(S, O, S)=S$, and $f_{E^{\prime}}(O, S, S)=O$. In other words, changing the set of evaluees changes the overall scores assigned by $f$ to the profiles $(S, O, S)$ and $(O, S, S)$. Thus, $f$ is not independent. This means that an evaluator who wished to use $f$ would need to have some mechanism for ascertaining the evaluees' profile orderings. Moreover, as the example illustrates, misrepresentation of profile orderings - and even the influence of unintentional biases could have a significant impact on the outcome of the evaluation. This phenomenon is analogous to the problem of insincere or strategic voting in social choice theory.

It is also worth noting that, while $f$ may be equitable with respect to $E$ (in this example, the profile orderings of $a, b$, and $c$ are not specified completely enough to make a definitive conclusion), $f$ is certainly not equitable with respect to $E^{\prime}$; in particular, $w$ perceives inequity since $(S, S, O) \succ_{w}(S, O, S)$ and

$$
f_{E^{\prime}}(S, O, S)=S \succ U=f_{E^{\prime}}(S, S, O) .
$$

We leave it as an exercise to the reader to verify that $f$ is monotone and inclusive, but not consistent.

In the above examples, we considered rank aggregation functions that were monotone, but not necessarily equitable. In fact, because of the monotonicity requirement for profile orderings, the properties of monotonicity and equity (for rank aggregation functions) are closely related. This relationship can be made clear by examining the contrapositive of each property:

- Monotonicity: For all $x, y \in X$ and all $E \in \mathcal{P}(\mathcal{E}), f_{E}(y) \succ f_{E}(x)$ implies $x \ngtr y$.

- Equity: For all $x, y \in X$, all $E \in \mathcal{P}(\mathcal{E})$, and all $e \in E, f_{E}(y) \succ f_{E}(x)$ implies $y \succeq_{e} x$.

The difference between monotonicity and equity therefore amounts to the difference between the conditions of $x \ngtr y$ and $y \succeq_{e} x$. If $y \gg x$, then it is certainly the case that $y \succeq_{e} x$ for all $e$. But, as we have seen, $x \ngtr y$ does not necessarily imply $y \gg x$. Likewise, it is also possible for $x \gg y$ and $y \succeq_{e} x$, which happens if and only if $e$ is indifferent between $x$ and $y$.

The arguments for monotonicity and equity are often similar, and so we will sometimes invoke the following lemma to prove both properties simultaneously:

Lemma 2.6. Let $f$ be a rank aggregation function, and suppose that for all $x, y \in X$, $f_{E}(y) \succ f_{E}(x)$ implies both (1) $x \ngtr y$, and (2) $y \succeq_{e} x$ for every evaluee $e$. Then $f$ is both monotone and (universally) equitable.

Finally, it is important to recognize the difference between universality — particularly, as it applies to the properties of consistency and equity - and independence. Independence requires the scores assigned to profiles to depend only on the profiles themselves, and not on any properties of the underlying set of evaluees. However, 
a rank aggregation function need not be independent to be universally consistent or equitable. The latter properties do not require the assigned scores to be invariant with respect to changes in the set of evaluees. Rather, these properties require only that the function be consistent/equitable with respect to each set of evaluees. Likewise, an independent rank aggregation function may be equitable with respect to some sets of evaluees, but not others. On the other hand, if an independent rank aggregation function is consistent with respect to any individual set of evaluees, then it will be universally consistent.

\section{Existence of ideal rank aggregation functions}

In the previous section, we saw an example of a rank aggregation function that was independent, monotone, consistent, and inclusive, but equitable (and thus ideal) only when $m=2$ (Example 2.4). The rank aggregation function in Example 2.5 fared even worse: it was monotone and inclusive, but not independent, consistent, or equitable. This, of course, begs the question: What would an ideal rank aggregation function look like for $m \geq 3$ ? Does such a function even exist? The next theorem provides a partial answer to this question.

Theorem 3.1. Let $m=3$, and define the rank aggregation function $f$ as

$$
f(x)= \begin{cases}z_{1} & \text { if } x=\vec{z}_{1}, \\ z_{3} & \text { if } x=\vec{z}_{3}, \\ z_{2} & \text { otherwise. }\end{cases}
$$

Then $f$ is an ideal rank aggregation function.

Proof. By definition, $f$ is independent and consistent. The argument for inclusivity is similar to that in Example 2.4.

For monotonicity and equity, we will use Lemma 2.6. So suppose $f(y) \succ f(x)$ for some $x, y \in X$. Then either $f(y)=z_{3}$ or $f(x)=z_{1}$. If the former, then $y=\vec{z}_{3}$ and $x \neq \vec{z}_{3}$. If the latter, then $x=\vec{z}_{1}$ and $y \neq \vec{z}_{1}$. In either case, $y \gg x$, which implies, by the monotonicity of profile orderings, that $y \succeq_{e} x$ for each evaluee $e$. Furthermore, $x \ngtr y$, and so $f$ is both monotone and equitable.

Unfortunately, the function from Theorem 3.1 cannot be extended to cases where $m \geq 4$. In fact, for $m \geq 4$, the properties of consistency and equity are incompatible.

Theorem 3.2. For $m \geq 4$, there does not exist a rank aggregation function that is consistent and equitable.

Proof. Let $m \geq 4$, and assume to the contrary that $f$ is a consistent and equitable rank aggregation function. Let $u=\left(z_{1}, z_{m}, z_{m}, \ldots, z_{m}\right)$, and let $E$ contain evaluees $e_{1}$ and $e_{2}$ for which

$$
\vec{z}_{2} \succ_{e_{1}} u \succ_{e_{2}} \vec{z}_{3}
$$


Since $f$ is equitable and consistent,

$$
z_{2}=f_{E}\left(\vec{z}_{2}\right) \succeq f_{E}(u) \succeq f_{E}\left(\vec{z}_{3}\right)=z_{3} .
$$

This, however, is a contradiction to the fact that $z_{3} \succ z_{2}$ by definition.

Theorem 3.2 shows that, for $m \geq 4$, it is impossible for a consistent rank aggregation function to be equitable with respect to every possible set of evaluees. However, it does not rule out the possibility of finding a consistent rank aggregation function that is equitable with respect to some sets of evaluees. If one also desires independence, then this distinction is moot. (If $f$ is independent, then since $f_{E}\left(\vec{z}_{2}\right) \succeq f_{E}\left(\vec{z}_{3}\right)$ for some set $E$ of evaluees, the same ordering would hold for every set of evaluees, leading again to a contradiction.) If we are willing to sacrifice independence, then we may have more options. We will explore this possibility further in Section 5.

\section{Cycles and uniqueness of ideal rank aggregation functions}

In the proof of Theorem 3.2, two evaluees' profile orderings were combined in a way that forced a contradiction under the assumptions of both consistency and equity. This idea can be generalized as follows.

Definition 4.1. Let $E \in \mathcal{P}(\mathcal{E})$. Suppose there exist $e_{1}, \ldots, e_{k} \in E$ and $x^{1}, \ldots, x^{k} \in X$ such that

$$
x^{1} \succ_{e_{1}} x^{2} \succ_{e_{2}} \cdots \succ_{e_{k-1}} x^{k} \succ_{e_{k}} x^{1} .
$$

Then the sequence $x^{1}, \ldots, x^{k}, x^{1}$ is said to be a strong $k$-cycle with respect to $E$.

The following theorem is immediate:

Theorem 4.2. Let $E \in \mathcal{P}(\mathcal{E})$, and let

$$
T_{E}=\{x \in X: x \text { belongs to some strong cycle with respect to } E\} .
$$

Let the relation $\sim_{E}$ on $T_{E}$ be defined by $x \sim_{E} y$ if and only $x$ and $y$ belong to a common strong cycle. Then $\sim_{E}$ is an equivalence relation on $T_{E}$.

The next theorem can be viewed as a generalization of Theorem 3.2.

Theorem 4.3. Let $E \in \mathcal{P}(\mathcal{E})$, and let $f$ be a rank aggregation function that is equitable with respect to $E$. If $x \sim_{E} y$ for some $x, y \in X$, then $f_{E}(x)=f_{E}(y)$.

Proof. Suppose $f$ is equitable, and let $x \sim_{E} y$ for some $x, y \in X$. Then $x$ and $y$ belong to a common strong $k$-cycle with respect to $E$ - that is,

$$
x \succ_{e_{1}} \cdots \succ_{e_{j-1}} y \succ_{e_{j}} \cdots \succ_{e_{k}} x
$$

for some $e_{1}, \ldots, e_{k} \in E$. But then equity requires that $f_{E}(x) \succeq f_{E}(y) \succeq f_{E}(x)$, which implies $f_{E}(x)=f_{E}(y)$. 
Two important corollaries follow.

Corollary 4.4. Let $E \in \mathcal{P}(\mathcal{E})$. If $\vec{z}_{i} \sim_{E} \vec{z}_{j}$ for some $z_{i}, z_{j} \in Z$ with $i \neq j$, then there does not exist a rank aggregation function that is both consistent and equitable with respect to $E$.

Proof. Suppose $f$ is equitable with respect to $E$, and suppose also that $\vec{z}_{i} \sim_{E} \vec{z}_{j}$ for some $z_{i}, z_{j} \in Z$ with $i \neq j$. By Theorem $4.3, f_{E}\left(\vec{z}_{i}\right)=f_{E}\left(\vec{z}_{j}\right)$. Since $i \neq j$, it is therefore impossible for $f$ to be consistent with respect to $E$.

Corollary 4.5. The function defined in Theorem 3.1 is the unique ideal rank aggregation function for $m=3$.

Proof. Let $m=3$, and let $f$ be an ideal rank aggregation function. Since $f$ is consistent, $f\left(\vec{z}_{1}\right)=z_{1}, f\left(\vec{z}_{2}\right)=z_{2}$, and $f\left(\vec{z}_{3}\right)=z_{3}$. Choose $x \in X$ such that $x \neq \vec{z}_{1}, \vec{z}_{2}, \vec{z}_{3}$. We will show that there exists $E \in \mathcal{P}(\mathcal{E})$ such that $x \sim_{E} \vec{z}_{2}$, which will imply (by Theorem 4.3) that $f(x)=f\left(\vec{z}_{2}\right)=z_{2}$. Consider three cases.

Case 1: $x \ngtr \vec{z}_{2}$ and $\vec{z}_{2} \ngtr x$. In this case, there exist evaluees $e_{1}$ and $e_{2}$ for which $\vec{z}_{2} \succ_{e_{1}} x \succ_{e_{2}} \vec{z}_{2}$. Let $E$ contain both $e_{1}$ and $e_{2}$. Then $x \sim_{E} \vec{z}_{2}$, as desired.

Case 2: $x \gg \vec{z}_{2}$. In this case, $x=\left(\left(\vec{z}_{3}\right)_{R},\left(\vec{z}_{2}\right)_{-R}\right)$ for some nonempty $R \subset C$. Let $y=\left(\left(\vec{z}_{1}\right)_{R},\left(\vec{z}_{3}\right)_{-R}\right)$. Note that (1) $\vec{z}_{2} \gg y$ and $y \gg \vec{z}_{2}$, and (2) $x \not>y$ and $y \gg x$. By assumption, $x \gg \vec{z}_{2}$. Thus, there exist evaluees $e_{1}, e_{2}$, and $e_{3}$ such that

$$
\vec{z}_{2} \succ_{e_{1}} y \succ_{e_{2}} x \succ_{e_{3}} \vec{z}_{2} .
$$

It follows that $x \sim_{E} \vec{z}_{2}$ for any $E \in \mathcal{P}(\mathcal{E})$ containing $e_{1}, e_{2}$, and $e_{3}$.

Case 3: $\vec{z}_{2} \gg x$. In this case, $x=\left(\left(\vec{z}_{1}\right)_{R},\left(\vec{z}_{2}\right)_{-R}\right)$ for some nonempty $R \subset C$. Let $y=\left(\left(\vec{z}_{3}\right)_{R},\left(\vec{z}_{1}\right)_{-R}\right)$. By a similar argument as in Case 2 , there exist evaluees $e_{1}, e_{2}$, and $e_{3}$ such that

$$
\vec{z}_{2} \succ_{e_{1}} x \succ_{e_{2}} y \succ_{e_{3}} \vec{z}_{2} .
$$

Thus, $x \sim_{E} \vec{z}_{2}$ for any $E \in \mathcal{P}(\mathcal{E})$ containing $e_{1}, e_{2}$, and $e_{3}$.

In each case, there exists $E \in \mathcal{P}(\mathcal{E})$ such that $x \sim_{E} \vec{z}_{2}$; hence $f_{E}(x)=f_{E}\left(\vec{z}_{2}\right)=z_{2}$. Since $f$ is independent, it follows that $f(x)=z_{2}$ for all $x \neq \vec{z}_{1}, \vec{z}_{2}, \vec{z}_{3}$. Thus, we have shown that $f$ is identical to the function from Theorem 3.1.

\section{Forfeiting independence}

As we saw in Section 3, it is impossible to define a consistent rank aggregation function for $m \geq 4$ that is equitable with respect to all possible sets of evaluees. In fact, Corollary 4.4 tells us that consistency and equity are compatible only when the set $E$ of evaluees is such that no two distinct uniform profiles belong to a common strong cycle. This necessary condition turns out to be sufficient as well, provided that we are willing to sacrifice independence. 
In order to proceed, we first need the following definition:

Definition 5.1. Let $E \in \mathcal{P}(\mathcal{E})$, and let $x, y \in X$. Suppose there exist $e_{1}, \ldots, e_{k} \in E$ and $x^{1}, \ldots, x^{k-1} \in X$ such that

$$
x \succ_{e_{1}} x^{1} \succ_{e_{2}} \cdots \succ_{e_{k-1}} x^{k-1} \succ_{e_{k}} y .
$$

Then $x$ is said to chain-dominate $y$ with respect to $E$, denoted $x \rightarrow_{E} y$.

Note that, for any $x, y \in X$, we have $x \sim_{E} y$ if and only if $x \rightarrow_{E} y$ and $y \rightarrow_{E} x$.

Theorem 5.2. Let $f$ be the rank aggregation function defined as follows:

$$
f_{E}(x)=\left\{\begin{array}{cl}
z_{1} & \text { if } x=\vec{z}_{1}, \\
\vdots & \text { if } f_{E}(x) \text { is not defined above and either } \max x=z_{i} \\
z_{i} & \text { or there exists } w \in X \text { such that } \max w=z_{i} \text { and } w \rightarrow_{E} x, \\
\vdots & \\
z_{m} & \text { otherwise. }
\end{array}\right.
$$

Then $f$ is monotone, inclusive, and equitable. Moreover, $f$ is consistent with respect to any $E \in \mathcal{P}(\mathcal{E})$ for which $\vec{z}_{i} \sim_{E} \vec{z}_{j}$ only if $i=j$.

Proof. For monotonicity, suppose $x \gg y$ for some $x, y \in X$. We must show that $f_{E}(x) \succeq f_{E}(y)$ for all $E \in \mathcal{P}(\mathcal{E})$. Suppose $f_{E}(x)=z_{i}$. If $i=1$, then $x=y=\vec{z}_{1}$, and so $f_{E}(x)=f_{E}(y)=z_{1}$. If $i=m$, then $f_{E}(x)=z_{m} \succeq f_{E}(y)$, as desired.

Now suppose $1<i<m$. Then either (1) $\max x=z_{i}$, or (2) there exists $w \in X$ with $\max w=z_{i}$ such that $w \rightarrow_{E} x$. If $\max x=z_{i}$, then $\max y \preceq z_{i}$, and so $f_{E}(y) \preceq z_{i}=f_{E}(x)$, as desired. Suppose, on the other hand, that there exists $w \in X$ with $\max w=z_{i}$ such that $w \rightarrow_{E} x$. Since $w \rightarrow_{E} x$, there exist $e_{1}, \ldots, e_{k} \in E$ and $x^{1}, \ldots, x^{k-1} \in X$ such that

$$
w \succ_{e_{1}} x^{1} \succ_{e_{2}} \cdots \succ_{e_{k-1}} x^{k-1} \succ_{e_{k}} x .
$$

But $x \gg y$, and so $x \succeq_{e_{k}} y$, which implies $x^{k-1} \succ_{e_{k}} y$. Thus $w \rightarrow_{E} y$, and so $f_{E}(y) \preceq z_{i}=f_{E}(x)$. In each case, $f_{E}(x) \succeq f_{E}(y)$. It follows that $f$ is monotone.

For inclusivity, let $R$ be any proper, nonempty subset of $C$. Then

$$
f_{E}\left(\vec{z}_{1}\right)=f_{E}\left(\left(\vec{z}_{1}\right)_{R},\left(\vec{z}_{1}\right)_{-R}\right)=z_{1},
$$

but

$$
f_{E}\left(\left(\vec{z}_{m}\right)_{R},\left(\vec{z}_{1}\right)_{-R}\right) \neq z_{1} .
$$

Since $f$ assigns different scores to two profiles that differ only on $R$, and $R$ was chosen arbitrarily, it follows that $C$ is minimal with respect to $f$. Thus, $f$ is inclusive.

For equity, let $E \in \mathcal{P}(\mathcal{E})$, and suppose $x \succ_{e} y$ for some $x, y \in X$ and some $e \in E$. We must show that $f_{E}(x) \succeq f_{E}(y)$. Let $f_{E}(x)=z_{i}$. If $i=1$, then $x=\vec{z}_{1}$, a contradiction to the fact that $x \succ_{e} y$. Therefore, $i>1$. If $i=m$, then $f_{E}(x)=z_{m} \succeq f_{E}(y)$, 
as desired. Now suppose $1<i<m$. By the definition of $f$, either $\max x=z_{i}$ or there exists $w \in X$ with $\max w=z_{i}$ such that $w \rightarrow_{E} x$. If $\max x=z_{i}$, then the fact that $x \rightarrow_{E} y$ (since $x \succ_{e} y$ ) implies, by the definition of $f$, that $f_{E}(y) \preceq z_{i}$. If, on the other hand, there exists $w \in X$ with $\max w=z_{i}$ such that $w \rightarrow_{E} x$, then it must be that $w \rightarrow_{E} y$ as well (since $x \succ_{e} y$ ), and so $f_{E}(y) \preceq z_{i}$. In either case, $f_{E}(x) \succeq f_{E}(y)$. It follows that $e$ cannot perceive inequity. Thus, $f$ is equitable with respect to $E$.

For consistency, let $E \in \mathcal{P}(\mathcal{E})$ such that $\vec{z}_{i} \sim_{E} \vec{z}_{j}$ only if $i=j$. It suffices to show that, for each $1<i \leq m$, there does not exist $w \in X$ such that $w \rightarrow_{E} \vec{z}_{i}$ and $\max w=z_{j}$ for some $j<i$. Suppose, to the contrary, that such a $w$ exists. Since $w \rightarrow{ }_{E} \vec{z}_{i}$, there exist $e_{1}, \ldots, e_{k} \in E$ and $x^{1}, \ldots, x^{k-1} \in X$ such that

$$
w \succ_{e_{1}} x^{1} \succ_{e_{2}} \cdots \succ_{e_{k-1}} x^{k-1} \succ_{e_{k}} \vec{z}_{i} .
$$

Moreover, since $\max w=z_{j}$ and $j<i$, it follows that $\vec{z}_{i} \gg \vec{z}_{j} \gg w$. By the monotonicity of $\succeq_{e_{1}}$, we have $\vec{z}_{i} \succeq_{e_{1}} \vec{z}_{j} \succeq_{e_{1}} w$. Since $w \succ_{e_{1}} x^{1}$, this implies that $\vec{z}_{i} \succ_{e_{1}} x^{1}$ and $\vec{z}_{j} \succ_{e_{1}} x^{1}$. By the monotonicity of $\succeq_{e_{k}}$, we have $\vec{z}_{i} \succeq_{e_{k}} \vec{z}_{j}$, and so $x^{k-1} \succ_{e_{k}} \vec{z}_{j}$. It follows that

$$
\vec{z}_{i} \succ_{e_{1}} x^{1} \succ_{e_{2}} \cdots \succ_{e_{k-1}} x^{k-1} \succ_{e_{k}} \vec{z}_{j} \succ_{e_{1}} x^{1} \succ_{e_{2}} \cdots \succ_{e_{k-1}} x^{k-1} \succ_{e_{k}} \vec{z}_{i} .
$$

But then $\vec{z}_{i} \sim_{E} \vec{z}_{j}$ with $i \neq j$, a contradiction. Therefore, it must be the case that $f_{E}\left(\vec{z}_{i}\right)=z_{i}$ for all $i$, and $f$ is consistent.

\section{Manifest inequity}

In our investigations up to this point, we have not made a distinction between the potential for inequity and the actual manifestation of inequity in the scores assigned by a given rank aggregation function. The former involves a systemic or structural concern - namely, that a rank aggregation function may lead to ratings that are perceived by some to be inequitable, regardless of whether any specific evaluee receives one of the profiles involved in these potential inequities. However, perceived inequity involving profiles that are actually assigned to evaluees - what we will call manifest inequity - is especially problematic. In this section, we will consider the more modest goal of avoiding manifest inequity, defined formally below.

Definition 6.1. Let $f$ be a rank aggregation function, and let $E \in \mathcal{P}(\mathcal{E})$. Suppose there exist $e_{1}, e_{2} \in E$ such that $x^{e_{1}} \succ_{e_{1}} x^{e_{2}}$ and $f_{E}\left(x^{e_{2}}\right) \succ f_{E}\left(x^{e_{1}}\right)$. Then

- $x^{e_{1}}$ and $x^{e_{2}}$ are called manifest profiles with respect to $E$; and

- $e_{1}$ is said to perceive manifest inequity.

A rank aggregation function for which no $e \in E$ perceives manifest inequity is said to be weakly equitable with respect to $E$. 
Clearly, one way to avoid manifest inequity is to limit the profiles that are actually assigned to evaluees. In situations in which a single evaluator both assigns profiles to evaluees and chooses the rank aggregation function, this solution is both simple and practical. In fact, we will show that it is possible to define a rank aggregation function that is independent, monotone, consistent, inclusive, and weakly equitable with respect to any set of evaluees that satisfies the pairwise dominance condition defined below.

Definition 6.2. A set $E$ of evaluees is said to be pairwise dominant if for all $e_{1}, e_{2} \in E$, either $x^{e_{1}} \gg x^{e_{2}}$ or $x^{e_{2}} \gg x^{e_{1}}$. Likewise, a set $S$ of profiles is said to be pairwise dominant if for all $x, y \in S$, either $x \gg y$ or $y \gg x$.

Theorem 6.3. Let $f$ be the rank aggregation function defined by $f(x)=\max x$. Then $f$ is independent, consistent, inclusive, monotone, and weakly equitable with respect to any pairwise dominant set $E$ of evaluees.

Proof. By definition, $f$ is independent and consistent. The proof of inclusivity and monotonicity is given in Example 2.4.

Now let $E$ be any pairwise dominant set of evaluees. We must show that $f$ is weakly equitable with respect to $E$. Suppose $x^{e_{1}} \succ_{e_{1}} x^{e_{2}}$ for some $e_{1}, e_{2} \in E$. Since $E$ is assumed to be pairwise dominant, either $x^{e_{1}} \gg x^{e_{2}}$ or $x^{e_{2}} \gg x^{e_{1}}$. If $x^{e_{2}} \gg x^{e_{1}}$, then the monotonicity of $\succeq_{e_{1}}$ implies $x^{e_{2}} \succeq_{e_{1}} x^{e_{1}}$, a contradiction. Therefore, $x^{e_{1}} \gg x^{e_{2}}$, and so

$$
f\left(x^{e_{1}}\right)=\max x^{e_{1}} \succeq \max x^{e_{2}}=f\left(x^{e_{2}}\right),
$$

as desired.

Theorem 6.3 raises two interesting combinatorial questions. First, what is the cardinality of a maximal pairwise dominant set of profiles? Second, how many such sets exist? The next theorem provides answers to these questions.

Theorem 6.4. Let $S$ be a maximal pairwise dominant set of profiles - i.e., a set of profiles $S$ that is pairwise dominant and is not a proper subset of any other pairwise dominant set. Then

$$
|S|=n(m-1)+1 .
$$

Moreover, the number of distinct pairwise dominant sets of profiles is equal to

$$
\frac{(n(m-1)) !}{(m-1) !^{n}} .
$$

Proof. First, note that $(X, \gg)$ is a poset, and $x$ covers $y$ in $(X, \gg)$ if and only if there exists $c \in C$ such that (1) $x_{i}=y_{i}$ for all $i \neq c$, and (2) $x_{c}=z_{k}$ and $y_{c}=z_{k-1}$ for some $1<k \leq m$. The cardinality of any maximal pairwise dominant set is the same as the length of a chain of maximum length in $(X, \gg)$. Such a chain must have maximum element $\vec{z}_{m}$, with each subsequent element formed by changing the 


\begin{tabular}{|rrrrr|}
\hline$n$ & $m$ & $m^{n}$ & $n(m-1)+1$ & $\frac{(n(m-1)) !}{(m-1) !^{n}}$ \\
\hline 3 & 3 & 27 & 7 & 90 \\
3 & 4 & 64 & 10 & 1680 \\
3 & 5 & 125 & 13 & 34650 \\
4 & 3 & 81 & 9 & 2520 \\
4 & 4 & 256 & 13 & 369600 \\
4 & 5 & 625 & 17 & 63063000 \\
\hline
\end{tabular}

Table 1. Combinatorial results for small $m, n$.

preceding element's score from $z_{k}$ to $z_{k-1}$ on exactly one criterion. Since there are $n$ criteria, and the score on each criterion may be decreased (from $z_{k}$ to $z_{k-1}$ ) exactly $m-1$ times before reaching $z_{1}$, it follows that a chain in $(X, \gg)$ can have length at most $n(m-1)+1$.

Each maximal chain (and, hence, each maximal pairwise dominant set) is uniquely determined by the order in which the criteria are decreased. Thus, each maximal pairwise dominant set corresponds to a sequence of $n(m-1)$ elements of $C$, where each of the $n$ elements of $C$ appears $m-1$ times. The standard formula for counting permutations with repetition thus implies that there are

$$
\frac{(n(m-1)) !}{(m-1) !^{n}}
$$

such sequences, as desired.

Table 1 illustrates the results of Theorem 6.4 for some small values of $m$ and $n$. While it is clear that pairwise dominant sets of profiles are small in comparison to the total number of profiles $\left(\mathrm{m}^{n}\right)$, it is also the case that there are many such sets to choose from. Therefore, an evaluator who wishes to take advantage of Theorem 6.3 must accept some fairly severe restrictions, but can satisfy these restrictions in a number of different ways.

\section{Summary and conclusions}

Rank disequilibrium can be a significant factor in social and intergroup conflict. In this article, we investigated rank disequilibrium in the context of multiple-criteria evaluation, using an axiomatic approach to show that, in general, it is impossible to define a function that aggregates scores on individual criteria while satisfying a relatively small set of desirable properties. In particular, we showed the notions of consistency and equity are generally incompatible. It seems perfectly reasonable to expect evaluees who receive the same score on every criterion to also receive that 
score as an overall rating. However, this apparently innocuous requirement opens the door for evaluees to perceive inequity.

The problems illuminated by our analysis are not insoluble. When evaluators are limited to only three possible scores (say, outstanding, satisfactory, and unsatisfactory), an ideal rank aggregation function can be found. However, the only such function assigns a overall rating of satisfactory to almost all profiles, rating evaluees as outstanding or unsatisfactory only when they receive these respective scores on every criterion.

Another potential solution is to allow the evaluator to use information about evaluees' individual profile orderings in order to assign scores that minimize the potential for perceived inequity. An evaluator who implements such a system is likely to be motivated more by political considerations than an actual concern for equity. Indeed, doing so requires a great deal of effort (to ascertain reliable information about evaluees' relative orderings of the various profiles) without an absolute guarantee that the ideals of consistency and equity will be achieved.

A more practical solution involves simply limiting the profiles assigned to evaluees to ensure that, for any pair of profiles assigned, one profile is viewed as more desirable by all rational evaluees (that is, all evaluees whose profile orderings meet the modest condition of monotonicity.) An evaluator need not have foreknowledge of the evaluees' views in order to adopt this strategy, but must be willing to possibly rate evaluees insincerely in order to avoid assigning profiles that fall outside the allowed set. She must also settle for the weaker goal of avoiding manifest inequity, rather than all perceived inequity. Broader systemic or structural concerns may still persist, but the most glaring perceptions of inequity will be eliminated.

Our investigations rest on several assumptions that could be relaxed in future work. For example, we have required the same rating scale (i.e., the same set of scores) to be used for each criterion, as well as for the overall evaluation. We have also assumed the set of scores to be discrete. In other multiple-criteria decision contexts (such as voting on referenda; see [Bradley et al. 2005], for example), the distinction between discrete and continuous alternative sets has been shown to be significant.

Our model does not incorporate any assumptions about the relative importance of the evaluation criteria, nor does it address potential interdependencies among criteria. (In early critiques of research on rank disequilibrium, Doreian and Stockman [1969] and Hartman [1974] raise related concerns.) Given the importance of separability in economics and social choice theory [Bradley et al. 2005; Gorman 1968; Hodge and Schwallier 2006], these areas would seem to warrant further investigation.

\section{References}

[Bradley et al. 2005] W. J. Bradley, J. K. Hodge, and D. M. Kilgour, "Separable discrete preferences", Math. Social Sci. 49:3 (2005), 335-353. MR Zbl 
[Doreian and Stockman 1969] P. Doreian and N. Stockman, "A critique of the multidimensional approach to stratification”, Sociol. Rev. 17:1 (1969), 47-65.

[Engel 1988] U. Engel, "Status inconsistency and criss-cross in an adolescent society", Int. Sociol. 3:3 (1988), 283-300.

[Evan and Simmons 1969] W. M. Evan and R. G. Simmons, "Organizational effects of inequitable rewards: two experiments in status inconsistency”, Adm. Sci. Quart. 14:2 (1969), 224-237.

[Galtung 1964] J. Galtung, "A structural theory of aggression”, J. Peace Res. 1:2 (1964), 95-119.

[Gorman 1968] W. M. Gorman, "The structure of utility functions", Rev. Econ. Stud. 35:4 (1968), 367-390.

[Hartman 1974] M. Hartman, "On the definition of status inconsistency", Amer. J. Sociol. 80:3 (1974), $706-721$.

[Hernes 1969] G. Hernes, "On rank disequilibrium and military coups d'etat”, J. Peace Res. 6:1 (1969), 65-72.

[Hodge and Schwallier 2006] J. K. Hodge and P. Schwallier, "How does separability affect the desirability of referendum election outcomes?", Theory Decis. 61:3 (2006), 251-276. MR Zbl

[Kriesberg 1998] L. Kriesberg, Constructive conflicts: from escalation to resolution, Rowman \& Littlefield, Lanham, MD, 1998.

[Muller and Jukam 1983] E. N. Muller and T. O. Jukam, "Discontent and aggressive political participation", Br. J. Political Sci. 13:2 (1983), 159-179.

[Pruitt and Kim 2004] D. G. Pruitt and S. H. Kim, Social conflict: escalation, stalemate, and settlement, McGraw Hill, New York, NY, 2004.

[Segal et al. 1970] D. R. Segal, M. W. Segal, and D. Knoke, "Status inconsistency and self-evaluation", Sociom. 33:3 (1970), 347-357.

Received: 2015-10-16 Revised: 2015-12-17 Accepted: 2015-12-25

hodgejo@gvsu.edu

Department of Mathematics, Grand Valley State University, Allendale, MI 49401, United States

fayest@gmail.com

Mount Holyoke College, South Hadley, MA 01075, United States

jamie.woelk@gmail.com

Western State Colorado University, Gunnison, CO 81231, United States 


\section{involve}

msp.org/involve

\section{INVOLVE YOUR STUDENTS IN RESEARCH}

Involve showcases and encourages high-quality mathematical research involving students from all academic levels. The editorial board consists of mathematical scientists committed to nurturing student participation in research. Bridging the gap between the extremes of purely undergraduate research journals and mainstream research journals, Involve provides a venue to mathematicians wishing to encourage the creative involvement of students.

\section{MANAGING EDITOR}

Kenneth S. Berenhaut Wake Forest University, USA

\section{BOARD OF EDITORS}

Colin Adams Williams College, USA

John V. Baxley

Arthur T. Benjamin

Martin Bohner

Nigel Boston

Amarjit S. Budhiraja

Pietro Cerone

Scott Chapman

Joshua N. Cooper

Jem N. Corcoran

Toka Diagana

Michael Dorff

Sever S. Dragomir Behrouz Emamizadeh Joel Foisy

Errin W. Fulp

Joseph Gallian

Stephan R. Garcia

Anant Godbole

Ron Gould

Andrew Granville

Jerrold Griggs

Sat Gupta

Jim Haglund

Johnny Henderson

Jim Hoste

Natalia Hritonenko

Glenn H. Hurlbert

Charles R. Johnson

K. B. Kulasekera

Gerry Ladas
Wake Forest University, NC, USA

Harvey Mudd College, USA

Missouri U of Science and Technology, USA

University of Wisconsin, USA

U of North Carolina, Chapel Hill, USA

La Trobe University, Australia

Sam Houston State University, USA

University of South Carolina, USA

University of Colorado, USA

Howard University, USA

Brigham Young University, USA

Victoria University, Australia

The Petroleum Institute, UAE

SUNY Potsdam, USA

Wake Forest University, USA

University of Minnesota Duluth, USA

Pomona College, USA

East Tennessee State University, USA

Emory University, USA

Université Montréal, Canada

University of South Carolina, USA

U of North Carolina, Greensboro, USA

University of Pennsylvania, USA

Baylor University, USA

Pitzer College, USA

Prairie View A\&M University, USA

Arizona State University,USA

College of William and Mary, USA

Clemson University, USA

University of Rhode Island, USA
Suzanne Lenhart

Chi-Kwong Li

Robert B. Lund

Gaven J. Martin Mary Meyer

Emil Minchev

Frank Morgan

Mohammad Sal Moslehian

Zuhair Nashed

Ken Ono

Timothy E. O'Brien

Joseph O'Rourke

Yuval Peres

Y.-F. S. Pétermann

Robert J. Plemmons

Carl B. Pomerance

Vadim Ponomarenko

Bjorn Poonen

James Propp

Józeph H. Przytycki

Richard Rebarber

Robert W. Robinson

Filip Saidak

James A. Sellers

Andrew J. Sterge

Ann Trenk

Ravi Vakil

Antonia Vecchio

Ram U. Verma

John C. Wierman

Michael E. Zieve
University of Tennessee, USA

College of William and Mary, USA

Clemson University, USA

Massey University, New Zealand

Colorado State University, USA

Ruse, Bulgaria

Williams College, USA

Ferdowsi University of Mashhad, Iran

University of Central Florida, USA

Emory University, USA

Loyola University Chicago, USA

Smith College, USA

Microsoft Research, USA

Université de Genève, Switzerland

Wake Forest University, USA

Dartmouth College, USA

San Diego State University, USA

UC Berkeley, USA

U Mass Lowell, USA

George Washington University, USA

University of Nebraska, USA

University of Georgia, USA

U of North Carolina, Greensboro, USA

Penn State University, USA

Honorary Editor

Wellesley College, USA

Stanford University, USA

Consiglio Nazionale delle Ricerche, Italy University of Toledo, USA

Johns Hopkins University, USA

University of Michigan, USA

PRODUCTION

Silvio Levy, Scientific Editor

Cover: Alex Scorpan

See inside back cover or msp.org/involve for submission instructions. The subscription price for 2017 is US $\$ 175 /$ year for the electronic version, and $\$ 235$ /year $(+\$ 35$, if shipping outside the US) for print and electronic. Subscriptions, requests for back issues from the last three years and changes of subscribers address should be sent to MSP.

Involve (ISSN 1944-4184 electronic, 1944-4176 printed) at Mathematical Sciences Publishers, 798 Evans Hall \#3840, c/o University of California, Berkeley, CA 94720-3840, is published continuously online. Periodical rate postage paid at Berkeley, CA 94704, and additional mailing offices.

Involve peer review and production are managed by EditFLOW ${ }^{\circledR}$ from Mathematical Sciences Publishers.

PUBLISHED BY

mathematical sciences publishers

nonprofit scientific publishing

http://msp.org/

(C) 2017 Mathematical Sciences Publishers 


\section{involve 2017 vol. $10 \quad$ no. 1}

Intrinsically triple-linked graphs in $\mathbb{R} P^{3}$

JARED Federman, Joel FoISY, KRISTIN MCNAMARA AND EMILY

STARK

A modified wavelet method for identifying transient features in time signals with applications to bean beetle maturation

DaVid McMorris, PaUl PEARSON AND BRIAN YURK

A generalization of the matrix transpose map and its relationship to the twist of the polynomial ring by an automorphism

ANDREW MCGinnis AND Michaela VANClifF

Mixing times for the rook's walk via path coupling

Cam Mcleman, Peter T. Otto, John Rahmani and Matthew

SUTTER

The lifting of graphs to 3-uniform hypergraphs and some applications to hypergraph Ramsey theory

Mark Budden, Josh Hiller, Joshua Lambert And Chris SANFord

The multiplicity of solutions for a system of second-order differential equations

Olivia Bennett, Daniel Brumley, BRitney Hopkins, Kristi

KARbER AND ThOMAS Milligan

Factorization of Temperley-Lieb diagrams

Dana C. Ernst, Michael G. Hastings and Sarah K. Salmon

Prime labelings of generalized Petersen graphs

Steven A. Schluchter, Justin Z. Schroeder, Kathryn Cokus,

RYAN ELLINGSON, HAYLEY HARRIS, ETHAN RARITY AND THOMAS

WILSON

A generalization of Zeckendorf's theorem via circumscribed $m$-gons

Robert Dorward, Pari L. Ford, Eva Fourakis, Pamela E. Harris,

Steven J. Miller, Eyvindur Palsson and Hannah Paugh

Loewner deformations driven by the Weierstrass function

JOAN LIND AND JESSICA ROBINS

Rank disequilibrium in multiple-criteria evaluation schemes

Jonathan K. Hodge, Faye Sprague-Williams and Jamie Woelk 